\begin{tabular}{|c|l|}
\hline Title & Excitonic spin-state preservation mediated by optical-phonon resonant excitation in a single quantum dot \\
\hline Author(s) & Kumano, H.; Kobay ashi, H.; Ekuni, S.; Hay ashi, Y.; Jo, M.; Sasakura, H.; A dachi, S.; Muto, S.; Suemune, I. \\
\hline Citation & $\begin{array}{l}\text { Physical Review. B, Condensed Matter and Materials Physics, 78(8), 081306 } \\
\text { https:/doi.org/_0.1103PhysRevB.78.081306 }\end{array}$ \\
\hline Issue Date & 2008_08-25 \\
\hline Doc URL & http://hdl.handle.net/2115/34638 \\
\hline Rights & ○ 2008 A merican Physical Society \\
\hline Type & article \\
\hline File Information & 08_Kumano_PRB.pdf \\
\hline
\end{tabular}

Instructions for use 


\title{
Excitonic spin-state preservation mediated by optical-phonon resonant excitation in a single quantum dot
}

\author{
H. Kumano, ${ }^{1,2}$ H. Kobayashi, ${ }^{1}$ S. Ekuni, ${ }^{1}$ Y. Hayashi, ${ }^{1}$ M. Jo, ${ }^{1}$ H. Sasakura, ${ }^{1}$ S. Adachi, ${ }^{3}$ S. Muto, ${ }^{3}$ and I. Suemune ${ }^{1,2}$ \\ ${ }^{1}$ RIES, Hokkaido University, Sapporo 001-0021, Japan \\ ${ }^{2}$ Japan Science and Technology Corporation (CREST), Saitama 332-0012, Japan 332-0012, Japan \\ ${ }^{3}$ Graduate School of Engineering, Hokkaido University, Sapporo, 060-8628, Japan
}

(Received 4 June 2008; revised manuscript received 14 July 2008; published 25 August 2008)

\begin{abstract}
High degree of excitonic spin-state preservation during energy relaxation is demonstrated in a single quantum dot. Optical-phonon resonances and corresponding suppression of spin relaxation are clearly identified as dip structures in photoluminescence excitation spectra probed by the positive trion emission. Consequently, under the longitudinal optical-phonon resonant excitation, a distinguishably high degree of circular polarization up to $\sim 0.85$ is achieved. Extended spin-relaxation time in the positive trion ground state compared to that in neutral exciton ground state is also revealed.
\end{abstract}

DOI: 10.1103/PhysRevB.78.081306

PACS number(s): 78.67.Hc, 71.35.- y, 72.25.Fe, 72.25.Rb

Spin states in a single quantum dot (QD) can be one of the most fundamental physical platform for quantum bits (qubits) since the spin states in a QD can be much more stabilized than those in higher dimensional systems. ${ }^{1}$ They are also promising from the viewpoint of realizing practical solid-state devices and their integrations based on highly developed semiconductor technologies. Another essential feature of electronic spin states is the ability to mutually convert into photon polarization states or vise versa via dipole interactions. This enables us to prepare QD-based nonclassical Fock-state photons with well-defined polarizations, which are required in quantum key distribution. ${ }^{2}$

In order to realize efficient state conversions between exciton spins in a QD and the photon polarizations in number states, exciton spins should be highly preserved in each process of the state conversions. Toward this direction, spinrelaxation mechanisms in a QD have been widely investigated in terms of the electron-hole $(e-h)$ exchange interaction, ${ }^{3,4}$ the spin-orbit interaction, ${ }^{5-7}$ as well as the hyperfine interaction ${ }^{8-11}$ for both neutral excitons and singly charged excitons (trions). Among them, trions are free from the $e-h$ exchange interaction ${ }^{4}$ and this makes them more attractive than neutral excitons $\left(X^{0}\right)$. Depolarization of photoexcited electron spins during energy relaxation is generally much slower than hole spins, ${ }^{12}$ which makes positively charged trions $\left(X^{+}\right)$with spin-singlet hole pairs more attractive than negatively charged trions $\left(X^{-}\right)$. Furthermore, since the initial and final states of the $X^{+}$emission are half spin systems, both states are spin degenerate due to Kramer's theorem in the absence of a magnetic field. Thus, the $X^{+}$state couples to the degenerate two kinds of photons with orthogonal circular polarizations. ${ }^{13}$ Therefore, the $X^{+}$state is an excellent spin state to examine the state conversions between the exciton spins in a QD and the photon polarizations.

Experimentally, the relaxation of the spin states can be directly examined by measuring the degree of circular polarization (DCP) of photons emitted from a QD under circularly polarized photoexcitation. In actual experimental setups, measurements under the resonant excitation of exciton ground states are rather difficult due to the leakage photons from the exciting laser sources. Therefore, the spin relaxation during energy relaxation processes is mostly involved in the measured DCP. ${ }^{14-16}$ Toward the ideal quantum-state conversions between exciton spin states and photon polarizations, quantitative understanding of both the spin relaxation during the energy relaxation and the subsequent one between spindegenerate trion ground states in a single QD is required.

In this Rapid Communication, stability of photogenerated exciton spin during and after energy relaxation is studied with detailed measurements of polarization-selective photoluminescence excitation (PLE) spectra and the relevant DCP. The contribution of the optical-phonon resonance for the preservation of the spin states is clearly identified as dip structures in the polarization-selective PLE spectra measured under the cross-circular detection with respect to the excitation polarization. High DCP up to $\sim 0.85$ is demonstrated without external magnetic field. Extended spin-relaxation time in the $X^{+}$ground state compared to that in $X^{0}$ ground state is revealed and the influence of the dynamic nuclear polarization (DNP) is also discussed.

$\mathrm{In}_{0.75} \mathrm{Al}_{0.25} \mathrm{As}$ QDs were grown on a semi-insulating (001)-GaAs substrate by molecular beam epitaxy and the details of this sample preparation are described in Ref. 17. After the growth, the sample was etched into mesa structures with diameters of $\sim 150 \mathrm{~nm}$ for isolating single QD from the dot ensemble with the density of around $5 \times 10^{10}$ dots $/ \mathrm{cm}^{2}$. For a single dot spectroscopy, the sample was kept at $20 \mathrm{~K}$. A continuous-wave Ti:sapphire laser was used as a circularly polarized excitation source. An objective lens with the numerical aperture of 0.42 focused the laser beam on one of the mesa structures and collected photoluminescence (PL) emitted from the mesa. Collected luminescence was dispersed by a 0.64-m triple monochromator and was introduced into a liquid-nitrogen cooled $\mathrm{Si}$ charge-coupled-device detector. The PL polarization was analyzed with $\sigma^{+} / \sigma^{-}$detection employing a set of quarter-wave plate and a fixed GlanThomson linear polarizer in front of the monochromator, where $\sigma^{+}\left(\sigma^{-}\right)$denotes the circular polarization with a helicity of $+1(-1)$. The overall system resolution was $4.5 \mu \mathrm{eV}$.

PL spectra measured from a single QD under the specified excitation energies (a)-(d) are shown in Fig. 1. When the excitation energy is above $1.67 \mathrm{eV}$ corresponding to the 


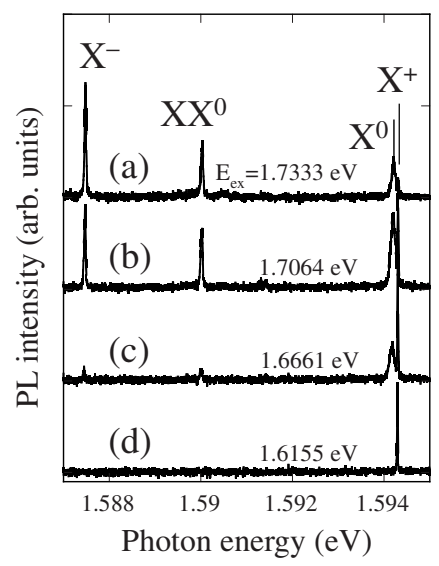

FIG. 1. PL spectrum from a single QD under four excitation energies (a)-(d). For above WL excitation (a) and (b), positively charged trion $\left(X^{+}\right)$, neutral exciton $\left(X^{0}\right)$, neutral biexciton $\left(X X^{0}\right)$, and negatively charged trion $\left(X^{-}\right)$are observed.

wetting-layer (WL) absorption edge, four emission lines originating from $X^{+}, X^{0}, X^{-}$, and neutral biexciton $\left(X X^{0}\right)$ were dominantly observed, which were assigned carefully by several independent measurements. ${ }^{18}$ With lowering the excitation energy below that of the WL, excitonic species composed of twin electrons systematically faded away and finally almost exclusive population of the $X^{+}$state was observed as displayed in Fig. 1(d). This is possible because, in this experiment, the specific QD was selected which involves a residual hole on average before the subsequent intradot photoexcitation, which creates pair(s) of electron and hole.

Figure 2(a) shows the polarization-selective PLE spectra measured under the $\sigma^{+}$excitation and were shown as a function of the excitation energy relative to the detected $X^{+}$emis-
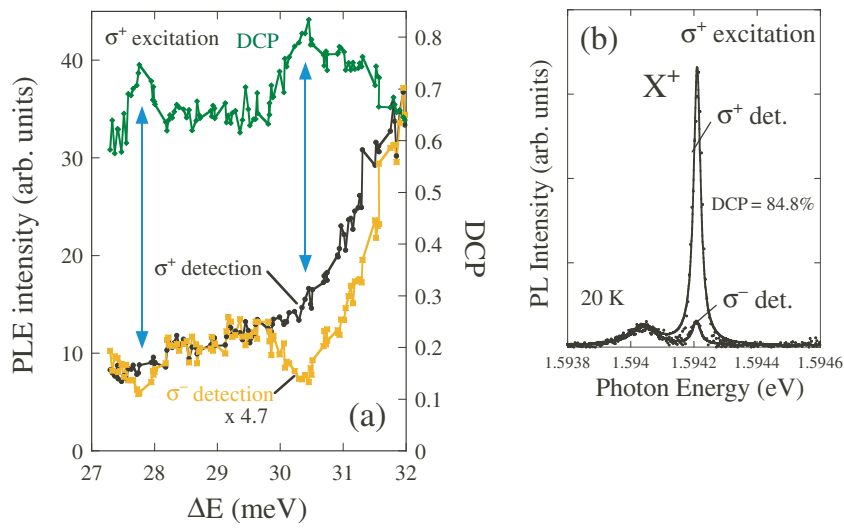

FIG. 2. (a) (Color online) Polarization-selective PLE spectra under $\sigma^{+}$excitation. Excitation energy is measured from the $X^{+}$ emission energy. The PLE spectrum with $\sigma^{-}$detection is multiplied by 4.7. Peak structures in DCP and corresponding dip structures in PLE indicated by arrows are attributed to InAs LO- $(30.5 \mathrm{meV})$ and TO- (27.4 meV) phonon resonances. (b) Polarization-dependent PL spectra under InAs LO-phonon resonant excitation. DCP amounts to $\sim 0.85$ for the $X^{+}$emission without external magnetic field. The small peak which appeared at the photon energy of $1.5932 \mathrm{eV}$ is the $X^{0}$ emission $(\mathrm{DCP}<0.05)$. sion line $(1.59421 \mathrm{eV})$. Here the energy resolution of the excitation energy was about $50 \mu \mathrm{eV}$. Excitation energy investigated was located at slightly lower region of Fig. 1(d). The PLE spectrum measured with the $\sigma^{-}$detection (hereafter designated as $\sigma^{-}$-PLE) was multiplied by 4.7 to clearly show the spectral difference to the $\sigma^{+}$detection $\left(\sigma^{+}\right.$-PLE). The DCP defined by $\left(I^{+}-I^{-}\right) /\left(I^{+}+I^{-}\right)$, where $I^{+}\left(I^{-}\right)$denotes the PLE intensity measured in the $\sigma^{+}\left(\sigma^{-}\right)$polarization, is also shown in Fig. 2(a). The multiplication factor of 4.7 given above for the $\sigma^{-}$-PLE corresponds to the average DCP of 0.65 measured under the nonresonant condition. Two peaks observed in the DCP almost agree with the energies of InAs longitudinal optical (LO)- (30.5 meV) and transverse optical (TO)- $(27.4 \mathrm{meV})$ phonon resonances. ${ }^{19}$ The comparison of two PLE spectra detected with orthogonal polarizations shows that the suppressed $\sigma^{-}$-PLE intensity contributes to these DCP peaks near the LO and TO resonances, and these results were essentially unchanged under the reversed polarization configuration.

In general, the polarization-selective PLE spectra involve spin flips experienced both during and after energy relaxation processes to the $X^{+}$state. These two processes are separately studied because the latter, i.e., spin flips between the two spin-degenerate $X^{+}$ground states-occurring after $X^{+}$ population with simultaneous excess phonon emission-are essentially independent of the excitation energy. The excitation-energy dependence of the polarization-selective PLE spectra predominantly reflects the spin flips during the energy relaxation. Thus, the dip structures at the LO- and TO-phonon energies observed in the $\sigma^{-}$-PLE spectrum are the direct evidence of the suppressed spin flips during the energy relaxation mediated by the optical phonons. In Fig. 2(a), the increase in the PLE intensities is observed above $\sim 31 \mathrm{meV}$, which is attributed to the onset of the continuum background transitions in the energy below the WL absorption edge. ${ }^{20}$ It is worth noting that, under the nonresonant excitation, the DCP still remains as high as 0.65 and shows almost constant except for the two optical-phonon resonances, although the simultaneous absorption of acoustic phonon can be expected. This observation indicates that the coupling between acoustic phonon and electron spin is less effective as a spin-relaxation mechanism, which is theoretically predicted in QDs. ${ }^{7}$

Polarization-dependent PL spectra measured under the InAs LO-phonon resonant excitation is shown in Fig. 2(b). Distinguishably high DCP up to $\sim 0.85$ is achieved for the $X^{+}$ emission without external magnetic field. This indicates that the spin polarization is highly stabilized by the InAs LOphonon resonant excitation.

In order to elucidate the spin flip in the entire processes involved after photoexcitation, a rate equation analysis was performed. In this model, a quantum dot with a residual hole state $\left|h^{+}\right\rangle$characterized by the angular-momentum projection $J_{z}= \pm 3 / 2$ was considered as depicted in the inset of Fig. 3 . Preferential virtual excitation of a pair of spin-down electron and spin-up hole by the $\sigma^{+}$excitation to the energy one LO phonon above the $X^{+}$state was dealt with, that is, opticalphonon-assisted resonant absorption in a single QD. ${ }^{21}$ Doubly degenerate $X^{+}$states are characterized by the angularmomentum projection $J_{z}= \pm 1 / 2$. Then, corresponding rate equations are given by 


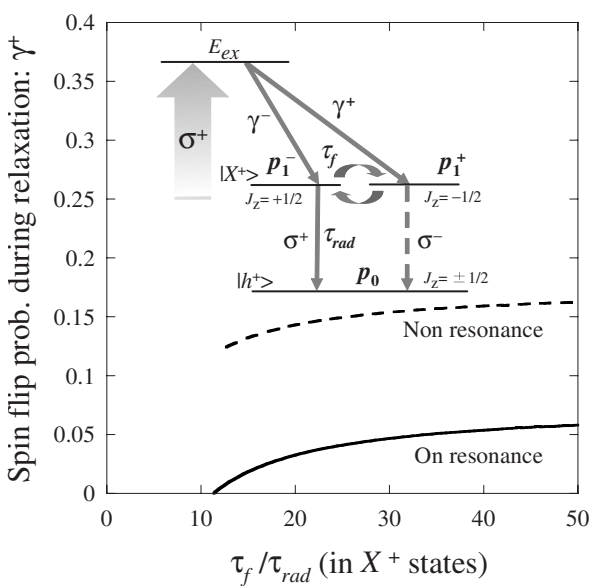

FIG. 3. Relations of two parameters characterizing spin flip during energy relaxation $\left(\gamma^{+}\right)$and in $X^{+}$ground states $\left(\tau_{f} / \tau_{\text {rad }}\right)$ for LO-phonon resonant (solid line) and nonresonant (dashed line) excitations, respectively. DCP values of 0.85 (on resonance) and 0.65 are used for the calculation. Other notations are given in the text. (Inset) Schematic of the model used for rate equation analysis for a QD with a residual hole. Spin flip both during and after energy relaxation process is included.

$$
\begin{gathered}
\frac{d p_{0}}{d t}=-\gamma^{+} G p_{0}-\gamma^{-} G p_{0}+\frac{p_{1}^{+}}{\tau_{\mathrm{rad}}}+\frac{p_{1}^{-}}{\tau_{\mathrm{rad}}}, \\
\frac{d p_{1}^{ \pm}}{d t}=\gamma^{ \pm} G p_{0}-\frac{p_{1}^{ \pm}}{\tau_{\mathrm{rad}}}-\frac{p_{1}^{ \pm}}{\tau_{f}}+\frac{p_{1}^{\mp}}{\tau_{f}},
\end{gathered}
$$

where $p_{0}$ is the probability of finding the system in the single hole states (Ref. 22), $p_{1}^{-}\left(p_{1}^{+}\right)$is the probability of finding the one in the trion $J_{z}=-1 / 2\left(J_{z}=+1 / 2\right)$ state with a spin-down (-up) unpaired electron, respectively, $G$ is the excitation rate, $\tau_{\text {rad }}$ is the radiative recombination lifetime of the trion, $\tau_{f}$ is the spin-flip time between the $J_{z}= \pm 1 / 2$ trion ground states, and $\gamma^{+}\left(\gamma^{-}\right)$is the spin-flip (preservation) probability during energy relaxation under the $\sigma^{+}$excitation, which satisfies $\gamma^{+}+\gamma^{-}=1$.

By solving the rate equations in steady-state condition, DCP is expressed as

$$
\mathrm{DCP}=\left(\gamma^{+}-\gamma^{-}\right)\left(\tau_{f} / \tau_{\mathrm{rad}}\right) /\left(\tau_{f} / \tau_{\mathrm{rad}}+2\right) .
$$

The relations of $\tau_{f} / \tau_{\text {rad }}$ and $\gamma^{+}$, which reproduce the experimentally observed DCP of 0.85 and 0.65 under the LOphonon resonant excitation and nonresonant excitation, respectively, are given by the solid and dashed lines in Fig. 3. The main points derived from this analysis are twofold: The first point is that the ratio of $\tau_{f} / \tau_{\text {rad }}$ should be larger than 11 . Since the radiative lifetime of the $X^{+}$state was measured to be $1.0 \mathrm{~ns},{ }^{18}$ this indicates the spin-flip time longer than $11 \mathrm{~ns}$. This ratio of $\tau_{f} / \tau_{\text {rad }}$ is larger than that of the neutral exciton of $\sim 3.6$ measured on the same QD reported in Ref. 18. This is partly because the $e$ - $h$ exchange interaction does not work in the trions. ${ }^{4}$

The second point is that the allowed range of the spin-flip probability $\gamma^{+}$during energy relaxation is given from Eq. (2) and Fig. 3. The insertion of infinity for the $\tau_{f} / \tau_{\text {rad }}$ ratio in Eq.

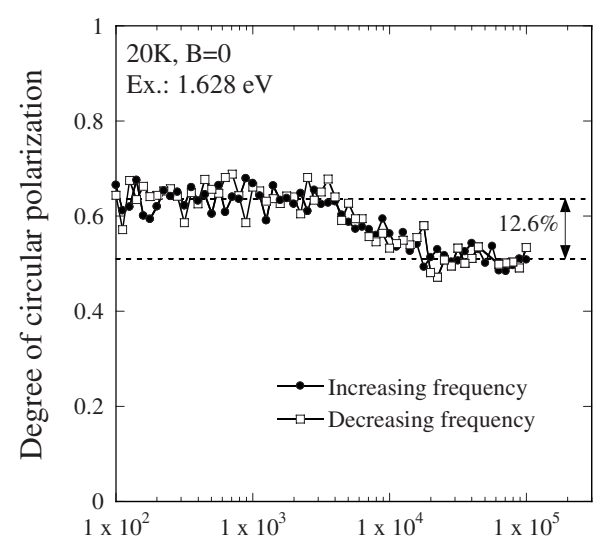

Polarization modulation frequency $(\mathrm{Hz})$

FIG. 4. Polarization modulation frequency dependence of the DCP in $X^{+}$line under $1.628 \mathrm{eV}$ excitation. The effect of the DNP on stabilizing the spin state was somewhat limited and estimated to be $\sim 13 \%$ in terms of DCP.

(2) gives the maximum spin-flip probabilities of 0.075 and 0.175 for the resonant and nonresonant excitations from the measured DCP of 0.85 and 0.65 , respectively. Since the allowed $\tau_{f} / \tau_{\text {rad }}$ ratio range is common for the both excitations, the lower limit of $\gamma^{+}$for the nonresonant excitation is given as 0.12 . Therefore, the spin-flip probability $\gamma^{+}$for the on- and off-optical-phonon resonant excitations is given by the ranges of $0-0.075$ and $0.12-0.175$, respectively. The spin state of $X^{+}$created with LO-phonon-assisted resonant excitation is shown to be highly stabilized in comparison to the nonresonant excitation.

In contrast to the $\gamma^{+}$parameter, the $\tau_{f} / \tau_{\text {rad }}$ ratio is inherent to the spin-flip mechanisms working on the trion ground states after energy relaxation. Under the steady-state circularly polarized excitation the nuclear magnetic field can be induced by spin-polarized electrons, ${ }^{11}$ which possibly modifies the $\tau_{f} / \tau_{\text {rad }}$ ratio. Actually, the Zeeman splitting of $\sim 7 \mu \mathrm{eV}$ was observed in the $X^{+}$emission line under the WL excitation (not shown), which corresponds to the magnetic field of $B_{N} \sim 0.33 \mathrm{~T}$ estimated with the $g$ factor of $g_{e}=$ $-0.37 .{ }^{23}$ In order to examine the influence of DNP on the observed high spin stability without an external magnetic field, the polarization modulation measurement of the DCP was performed and the results are shown in Fig. 4. In this experiment, alternate circularly polarized excitation was carried out using an electro-optical modulator ${ }^{24}$ and the modulation frequency was swept sequentially. As the modulation frequency was increased, the DCP started to decrease at $\sim 3 \mathrm{kHz}$ and then reached to the almost constant value above $20 \mathrm{kHz}$. This behavior was unaffected by the sweep direction. Above $20 \mathrm{kHz}$, where the DNP can no longer follow the modulation, the DCP still exhibits as high as 0.5. This indicates that the DNP has only a subsidiary contribution to the observed high DCP.

In conclusion distinguishably high degree of circular polarization up to $\sim 0.85$ was observed in a single quantum dot without external magnetic field by the optical-phonon mediated resonant excitation. Furthermore, the extension of the spin-relaxation time of the positive trion ground states by 
more than three times of the neutral excitons was demonstrated. The above findings are essential for implementing polarization-preserved single-photon emitter.

The authors would like to acknowledge Dr. H. Z. Song, $\mathrm{S}$. Hirose, and M. Takatsu for the sample preparation. This work was supported in part by the Grant-in-Aid for Scientific Research (S)(2), Grants No. 16106005; Young Scientists (A), No. 18681025; and by Hokkaido Innovation Through Nanotechnology Supports (HINTs) from the Ministry of Education, Culture, Sports, Science and Technology, Japan.
${ }^{1}$ E. Tsitsishvili, R. v. Baltz, and H. Kalt, Phys. Rev. B 67, 205330 (2003).

${ }^{2}$ N. Gisin, G. Ribordy, W. Tittel, and H. Zbinden, Rev. Mod. Phys. 74, 145 (2002).

${ }^{3}$ S. Laurent, B. Eble, O. Krebs, A. Lemaitre, B. Urbaszek, X. Marie, T. Amand, and P. Voisin, Phys. Rev. Lett. 94, 147401 (2005).

${ }^{4}$ G. V. Astakhov, A. V. Koudinov, K. V. Kavokin, I. S. Gagis, Y. G. Kusrayev, W. Ossau, and L. W. Molenkamp, Phys. Rev. Lett. 99, 016601 (2007).

${ }^{5}$ A. V. Khaetskii and Y. V. Nazarov, Phys. Rev. B 61, 12639 (2000).

${ }^{6}$ E. Tsitsishvili, R. v. Baltz, and H. Kalt, Phys. Rev. B 72, 155333 (2005).

${ }^{7}$ V. N. Golovach, A. Khaetskii, and D. Loss, Phys. Rev. Lett. 93, 016601 (2004).

${ }^{8}$ I. A. Merkulov, A. L. Efros, and M. Rosen, Phys. Rev. B 65, 205309 (2002).

${ }^{9}$ P.-F. Braun, X. Marie, L. Lombez, B. Urbaszek, T. Amand, P. Renucci, V. K. Kalevich, K. V. Kavokin, O. Krebs, P. Voisin, and Y. Masumoto, Phys. Rev. Lett. 94, 116601 (2005).

${ }^{10}$ B. Eble, O. Krebs, A. Lemaitre, K. Kowalik, A. Kudelski, P. Voisin, B. Urbaszek, X. Marie, and T. Amand, Phys. Rev. B 74, 081306(R) (2006).

${ }^{11}$ C. W. Lai, P. Maletinsky, A. Badolato, and A. Imamoglu, Phys. Rev. Lett. 96, 167403 (2006).

${ }^{12}$ S. Laurent, M. Senes, O. Krebs, V. K. Kalevich, B. Urbaszek, X. Marie, T. Amand, and P. Voisin, Phys. Rev. B 73, 235302 (2006).

${ }^{13}$ I. A. Akimov, T. Flissikowski, A. Hundt, and F. Henneberger, Phys. Status Solidi A 201, 412 (2004).

${ }^{14}$ A. Ebbens, D. N. Krizhanovskii, A. I. Tartakovskii, F. Pulizzi, T. Wright, A. V. Savelyev, M. S. Skolnick, and M. Hopkinson, Phys. Rev. B 72, 073307 (2005).

${ }^{15}$ M. Scheibner, G. Bacher, S. Weber, A. Forchel, T. Passow, and
D. Hommel, Phys. Rev. B 67, 153302 (2003).

${ }^{16}$ S. Mackowski, T. A. Nguyen, T. Gurung, K. Hewaparakrama, H. E. Jackson, L. M. Smith, J. Wrobel, K. Fronc, J. Kossut, and G. Karczewski, Phys. Rev. B 70, 245312 (2004).

${ }^{17}$ H. Sasakura, S. Adachi, S. Muto, H. Z. Song, T. Miyazawa, and T. Usuki, Jpn. J. Appl. Phys., Part 1 43, 2110 (2004).

${ }^{18}$ H. Kumano, S. Kimura, M. Endo, H. Sasakura, S. Adachi, S. Muto, and I. Suemune, J. Nanoelectron. Optoelectron. 1, 39 (2006)

${ }^{19}$ D. L. Stierwalt and R. F. Potter, Phys. Rev. 137, A1007 (1965).

${ }^{20}$ Y. Toda, O. Moriwaki, M. Nishioka, and Y. Arakawa, Phys. Rev. Lett. 82, 4114 (1999).

${ }^{21}$ A. Lemaître, A. D. Ashmore, J. J. Finley, D. J. Mowbray, M. S. Skolnick, M. Hopkinson, and T. F. Krauss, Phys. Rev. B 63, 161309(R) (2001).

${ }^{22}$ There have been reports that hole spin relaxation in the $J_{z}$ $= \pm 3 / 2$ states is extremely slow (Ref. 25). Our rate equation analysis predicted that the $X^{+}$emission shows strong saturation with the increase in the excitation power if the hole spin relaxation is slow as reported. Our additional experiments revealed that the polarization-selective $X^{+}$emission does not show saturation but grows linearly with the excitation. This indicates that the single hole spin relaxation is fast enough to average the $J_{z}$ $=+3 / 2$ and $J_{z}=-3 / 2$ state populations in our experiments.

${ }^{23}$ R. Kaji, S. Adachi, H. Sasakura, and S. Muto, Appl. Phys. Lett. 91, 261904 (2007).

${ }^{24}$ In order to deduce the DCP under alternate circularly polarized excitation, asymmetric duty ratio was used, i.e., duty ratio of $\sigma^{+}: \sigma^{-}=2: 1(1: 2)$ for measuring PL intensity $I \sigma^{+}\left(I \sigma^{-}\right)$with $\sigma^{+}\left(\sigma^{-}\right)$detection. Resultant circular polarization is defined as $\rho \equiv\left[\left(2 I \sigma^{+}+I \sigma^{-}\right)-\left(I \sigma^{+}+2 I \sigma^{-}\right)\right] /\left[\left(2 I \sigma^{+}+I \sigma^{-}\right)+\left(I \sigma^{+}+2 I \sigma^{-}\right)\right]$ $=\mathrm{DCP} / 3$. Accordingly, the DCP in Fig. 4 corresponds to $3 \rho$.

${ }^{25}$ B. D. Gerardot, D. Brunner, P. A. Dalgarno, P. Öhberg, S. Seidl, M. Kroner, K. Karrai, N. G. Stoltz, P. M. Petroff, and R. J. Warburton, Nature (London) 451, 441 (2008). 